Research Article

Published September 20, 2016

\title{
FLUOROQUINOLONE-RESISTANT ESCHERICHIA coli Infections After Transrectal Biopsy of the Prostate in the Veterans Affairs Healthcare System
}

\author{
AUTHORS \\ Elie A. Saade ${ }^{1,2}$, Nuntra Suwantarat ${ }^{1,2}$, Trina F. Zabarsky ${ }^{3}$, Brigid Wilson ${ }^{4}$, Curtis J. Donskey ${ }^{2,4}$

\section{AFFILIATED INSTITUTIONS} \\ ${ }^{1}$ Infectious Diseases Division, University Hospitals, Cleveland, Ohio \\ ${ }^{2}$ Case Western Reserve University School of Medicine, Cleveland, Ohio \\ ${ }^{3}$ Infection Control Department, Louis Stokes Cleveland VA Medical Center, Cleveland, Ohio \\ ${ }^{4}$ Geriatric Research Education and Clinical Center, Cleveland VA Medical Center, Cleveland, \\ Ohio
}

\section{STANDFIRST}

In the Veterans Affairs Health Care System, the incidence of Escherichia coli (E. coli) infection after transrectal biopsy has increased significantly since 2000 due to a dramatic rise in infections with fluoroquinolone-resistant $E$. coli.

\section{CORRESPONDING AUTHOR}

Elie A. Saade

Infectious Diseases Division

Basic Research Building Room 1031

Case Western Reserve University

Cleveland, OH, 44106

Phone: 216-286-6860

Fax: 216-844-1632

eliesaade2002@hotmail.com
DOI

10.20411/pai.v1i2.123 


\section{ABSTRACT}

Background: Recent reports suggest that infections due to fluoroquinolone-resistant Escherichia coli (E. coli) are an increasingly common complication of transrectal biopsy of the prostate (TBP) in the United States. A better understanding of the magnitude and scope of these infections is needed to guide prevention efforts. Our objective is to determine whether the incidence of infections due to fluoroquinolone-resistant $E$. coli after TBP has increased nationwide in the Veterans Affairs Health Care System and to identify risk factors for infection.

Methods: We performed a retrospective, observational cohort study and a nested case-control study within the US Deparment of Veterans Affairs Healthcare System. The primary outcomes were the incidence of urinary tract infection (UTI) and bacteremia with E. coli and with fluoroquinolone-resistant E. coli strains within 30 days after TBP. Secondary endpoints focused on the correlation between fluoroquinolone-resistance in all urinary E. coli isolates and post-TBP infection and risk factors for infection due to fluoroquinolone-resistant E. coli infection.

Results: 245,618 patients undergoing 302,168 TBP procedures from 2000 through 2013 were included in the cohort study, and 59,469 patients undergoing TBP from 2011 through 2013 were included in the nested case-control study. Between 2000 and 2013, there was a 5-fold increase in the incidence of E. coli UTI (0.18\%-0.93\%) and a 4-fold increase in the incidence of E. coli bacteremia $(0.04 \%-0.18 \%)$ after TBP that was attributable to an increase in the incidence of fluoroquinolone-resistant E. coli UTI $(0.03 \%-0.75 \%)$ and bacteremia $(0.01 \%-0.14 \%)$. The increasing incidence of fluoroquinolone-resistant $E$. coli infections after TBP occurred in parallel with increasing rates of fluoroquinolone-resistance in all urinary E. coli isolates. By multivariable logistic regression analysis, independent risk factors for fluoroquinolone-resistant $E$. coli UTI after TBP included diabetes mellitus, fluoroquinolone exposure, prior hospitalization, and prior cultures with fluoroquinolone-resistant gram-negative bacilli.

Conclusion: In the Veterans Affairs Healthcare System, the incidence of E. coli infection after TBP has increased significantly since 2000 due to a dramatic rise in infections with fluoroquinolone-resistant E. coli.

Keywords: bacteremia, case-control studies, cohort studies, early detection of cancer, Escherichia coli infections, microbial drug resistance, postoperative complications, prostate, retrospective studies, United States Department of Veterans Affairs, urinary tract infections

\section{INTRODUCTION}

An estimated 1 million American males undergo transrectal ultrasound-guided biopsy of the prostate (TBP) each year, usually for prostate cancer screening [1]. Because the biopsy needle passes through the rectal mucosa, the TBP procedure is associated with a significant risk of infectious complications, including urinary tract infection (UTI), prostatitis, and bacteremia [2-10]. Escherichia coli (E. coli) is the most common cause of these infections. In randomized trials, antibiotic prophylaxis has been shown to significantly reduce the frequency of infectious complications after TBP [11]. 
Fluoroquinolones are the antibiotics most often used for TBP peri-procedural prophylaxis in the United States $[11, \underline{12}]$. However, fluoroquinolone resistance in E. coli and other gram-negative bacilli (GNB) has increased steadily in recent years $[\underline{13}, \underline{14}]$, and there have been several reports of increasing rates of infection due to fluoroquinolone-resistant E. coli after TBP, mostly from individual institutions [ $[\underline{6}-\underline{9}, \underline{15}-\underline{19}]$. In 3 nonrandomized trials, modification of antimicrobial prophylaxis based on pre-procedure rectal culture results has been effective for prevention of infection due to fluoroquinolone-resistant E. coli after TBP [ $\underline{20}-\underline{22}]$. Modification of prophylaxis regimens for all patients undergoing TBP also has been effective [른 25$]$, but adverse consequences of this approach have been reported [26]. In order to prioritize efforts to develop effective strategies to prevent infections after TBP, there is a need for a better understanding of the magnitude and scope of these infections in the United States.

The Department of Veterans Affairs (VA) operates the largest healthcare system in the United States. E. coli is a common cause of infections in veterans, and fluoroquinolone-resistant strains, particularly sequence type 131, are widespread in the healthcare system [27]. Using national VA data for a 14-year period, we tested the hypothesis that the incidence of UTI and bacteremia with E. coli, particularly with fluoroquinolone-resistant E. coli strains, is increasing after TBP. We conducted a case-control study to identify risk factors for infection due to fluoroquinolone-resistant E. coli after TBP. We hypothesized that prior exposure to fluoroquinolones and or previous admission to the hospital would predict most cases of infection with fluoroquinolone-resistant $E$. coli after TBP.

\section{METHODS}

\section{Protection of Human Research Participants}

The institutional review board at the Cleveland VA Medical Center approved all study activities. Informed consent was waived.

\section{Study Design}

Using national VA databases, we determined the incidence of bacteriuria and bacteremia with $E$. coli and of fluoroquinolone-resistant E. coli within 30 days after TBP. Rates of post-TBP E. coli infections were compared over time and for different geographic regions. We examined the correlation between the percentage of fluoroquinolone-resistance for all urinary and blood E. coli and the incidence of post-TBP infection with fluoroquinolone-resistant E. coli post-TBP, both overall and by facility. A nested case-control study of patients undergoing TBP from 2011 through 2013 was conducted to identify risk factors for infection with fluoroquinolone-resistant E. coli.

\section{Data Sources and Definitions}

We used data initially generated by the VA Electronic Health Record at all 152 VA hospitals and 850 outpatient clinics. The Veterans Informatics and Computing Infrastructure (VINCI) was used to access and analyze data on demographics, laboratory, and microbiology results, comorbidities derived from the Veterans Health Administration Corporate Data Warehouse, and pharmacy data extracted from the Decision Support System, Pharmacy Benefits Management, and the Corporate Data Warehouse. Encrypted patient identifiers were used to linked data from different sources. 
We identified patients undergoing TBP in all VA hospitals and outpatient clinics in the VA Health Care System between January 1, 2000 and December 31, 2013 by searching for Current Procedural Terminology (CPT) code 55700, which designates "biopsy, prostate; needle or punch, single or multiple, any approach" and either CPT code 76872 for transrectal ultrasound or 76942 for ultrasonic guidance for the needle biopsy of the prostate. In parallel, we identified the subset of patients undergoing TBP with positive urine or blood cultures for E. coli within 30 days after TBP, and determined if the $E$. coli isolates were resistant to fluoroquinolones. We also determined the proportion of all E. coli urinary and blood isolates that were resistant to fluoroquinolones during the same time period.

Post-TBP bacteriuria and bacteremia were defined on the basis of a positive culture for E. coli within 30 days after TBP in urine or blood, respectively. For the purposes of the study, we classified E. coli isolates as fluoroquinolone-resistant if they were "intermediate" or "resistant" based on Clinical and Laboratory Standards Institute criteria for ciprofloxacin or, when not available, levofloxacin. Diabetes was defined based on the documentation of at least one of the appropriate International Classification of Diseases, Ninth Revision (ICD-9) codes at an inpatient VA encounter, or two separate outpatient visits within 12 months prior to the procedure, as described in the Veterans Aging Cohort Study methodology [28]. Prior admission to a VA inpatient facility within the previous year was defined based on documentation of a stay in an inpatient setting in the VA system within 365 days prior to the TBP procedure. Prior fluoroquinolone exposure was defined as a prescription for a fluoroquinolone antibiotic in the 365 days prior to the procedure, excluding the 90 days prior to the procedure because peri-procedural prophylaxis is typically prescribed $1-3$ months prior to the procedure.

\section{Statistical Analysis}

The yearly incidence of fluoroquinolone-resistant and fluoroquinolone-susceptible E. coli bacteriuria and bacteremia within 30 days after TBP was calculated per 100 procedures. Incidence of infections was compared over time and by VA-defined regions (Eastern, Western, Southern, and Central). For the period 2008 through 2013, we compared the facility-level incidence of postTBP infection with fluoroquinolone-resistant E. coli among facilities with differing prevalence of fluoroquinolone-resistance in urinary E. coli isolates (i.e., <20\%; 20\%-29.99\%; 30\%-39.99\%; and $\geq 40 \%)$.

We conducted a nested case-control study of TBP procedures performed between January 2011 and December 2013. For patients undergoing more than one TBP during this period, we only included the first procedure. Patients who had a bacteriuria with fluoroquinolone-resistant $E$. coli based on a positive urine culture within 30 days after TBP were compared with patients who did not have a urine culture positive for fluoroquinolone-resistant E. coli; patients with positive urine cultures for fluoroquinolone-susceptible E. coli bacteriuria were excluded from the analysis. Categoric variables were described using proportions and continuous variables were described using median and range. To identify risk factors for post-TBP infection with fluoroquinolone-resistant E. coli, we first conducted a univariate analysis to compare the probability of developing a bacteriuria in relation to the following factors: age at the time of the procedure, admission to a VA hospital within the previous year, history of isolation of fluoroquinolone-resistant E. coli from a urine culture within the previous year, diabetes mellitus, receipt of a systemic antibiotic within the previous year, and receipt of a fluoroquinolone within the previous year excluding the 90 days 
prior to the procedure. Fisher's exact test or chi-square test was used to test the strength of association among categoric variables as appropriate. Student's $t$-test was used to compare continuous variables. The statistical threshold for significance was set at $P=0.01$ for a 2-tailed test. Factors with a $P$-value less than 0.1 on a univariate analysis were placed into a multivariable model to determine the adjusted odds ratios. A $P$-value of 0.1 was selected rather than 0.2 because the small sample limited the number of variables that could be analyzed in a multivariable model. Finally, we calculated the proportion of fluoroquinolone-resistant $E$. coli bacteriuria that would have been detected by combinations of risk factors identified as statistically significant.

\section{RESULTS}

\section{E. coli Infections After TBP}

Between January 1, 2000 and December 31, 2013, a total of 302,168 TBP procedures were performed on 245,618 men from $121 \mathrm{VA}$ facilities. The number of TBP procedures performed per facility each year ranged from 1 to 848 (mean, 192; median, 158). Figure 1 shows respectively the incidence of post-TBP bacteriuria and bacteremia due to E. coli, stratified by fluoroquinolone-resistant and fluoroquinolone-susceptible isolates, and the concurrent yearly prevalence of fluoroquinolone resistance in all E. coli urinary or blood isolates. Overall, 1,936 (0.64\%) TBP procedures were complicated by E. coli bacteriuria and $502(0.17 \%)$ were complicated by $E$. coli bacteremia. Between 2000 and 2013, there was a 5-fold increase in the incidence of $E$. coli bacteriuria $(0.18 \%-0.93 \% ; P<0.0001)$ and a 4 -fold increase in the incidence of E. coli bacteremia $(0.04 \%-$ $0.18 \%$; $P<0.0001)$ after TBP. The incidence of post-TBP bacteriuria due to fluoroquinolone-susceptible E. coli remained stable during the study period $(0.15 \%$ and $0.18 \%$ in 2000 and 2013 , respectively; $P=0.5$ ), whereas the incidence of post-TBP bacteriuria due to fluoroquinolone-resistant $E$. coli increased by 25 -fold $(0.03 \%-0.75 \% ; P<0.0001)$, peaking in 2009 . The incidence of post-TBP bacteremia due to fluoroquinolone-susceptible E. coli remained stable during the study period $(0.04 \%$ and $0.03 \%$ in 2000 and 2013 , respectively; $P=0.9)$, whereas the incidence of post-TBP bacteremia due to fluoroquinolone-resistant $E$. coli increased by 23 -fold $(0.01 \%-0.14 \%$; $P<0.0001)$, peaking in 2012. During the study, the proportion of post-TBP E. coli isolates that were fluoroquinolone-resistant increased significantly for both urinary $(16.7 \%-80.4 \% ; P<0.0001)$ and bacteremia $(14.3 \%-80.6 \% ; P=0.002)$ isolates.

The increasing incidence of fluoroquinolone-resistant E. coli infections after TBP occurred in parallel with increasing rates of fluoroquinolone-resistance in all urinary and blood E. coli isolates (Figure 1). For 671,682 urinary E. coli isolates that were subjected to susceptibility testing during the study period, the proportion that was resistant to fluoroquinolones increased from $<10 \%$ in 2000 to $\sim 35 \%$ in 2008 , and subsequently remained stable. Similarly, the proportion of all blood $E$. coli isolates that was resistant to fluoroquinolones increased from less than $10 \%$ to $30 \%$ during the study period, peaking in 2011. Of note, results of susceptibility testing to ciprofloxacin and or levofloxacin were available for $97 \%$ of samples; when both were available, they were concordant in $99.5 \%$ of cases.

Figure 2 shows the increase of the incidence of post-TBP fluoroquinolone-resistant $E$. coli bacteriuria during the study period, stratified by region. The increase in the incidence of post-TBP bacteriuria occurred in all regions of the country. The yearly incidence of post-TBP fluoroquinolone-resistant E. coli bacteriuria varied considerably among VA facilities, ranging from $0 \%$ to $5.9 \%$. 
A

Fluoroquinolone-Resistant E. coli UTI Fluoroquinolone-Susceptible E. coli UTI

$\rightarrow-$ All Urine E. coli Fluoroquinolone Resistance

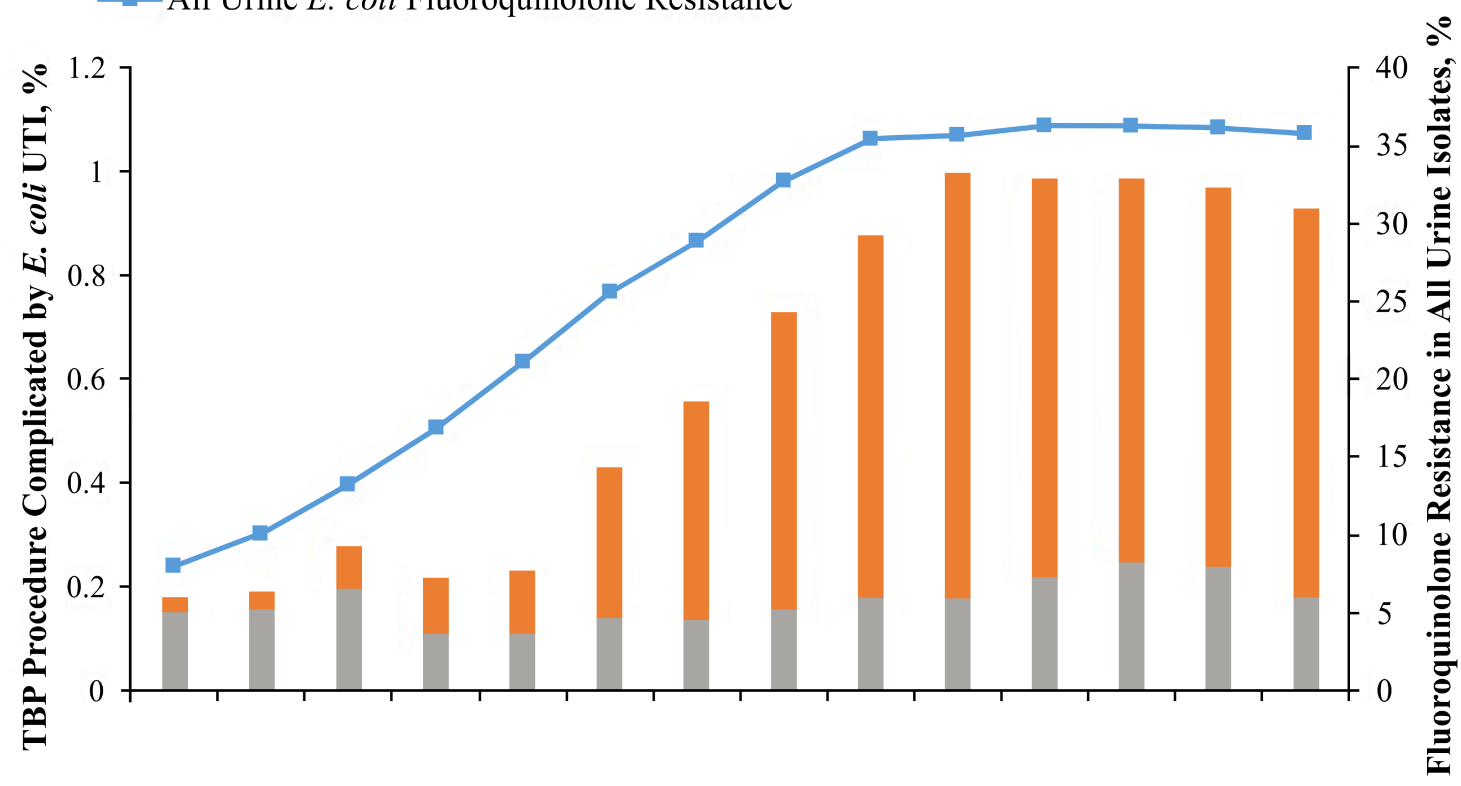

B

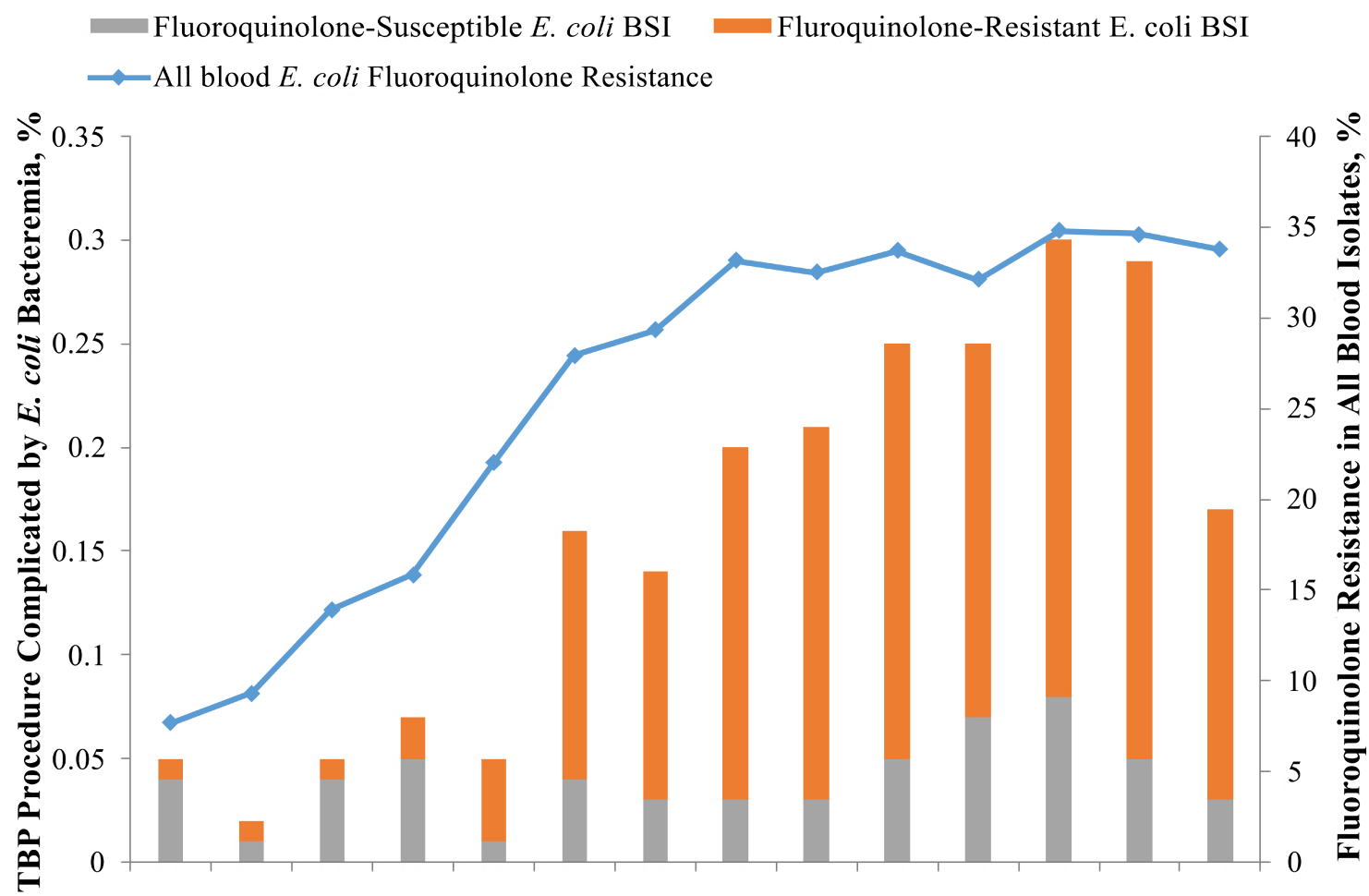

Figure 1. Escherichia coli Infections Following Transrectal Biopsy of the Prostate and Fluoroquinolone Resistance in All E. coli

Abbreviations: CI: confidence interval; FQ: fluoroquinolone; OR: odds ratio; TBP: transrectal biopsy of the prostate; UTI: urinary tract infection. 


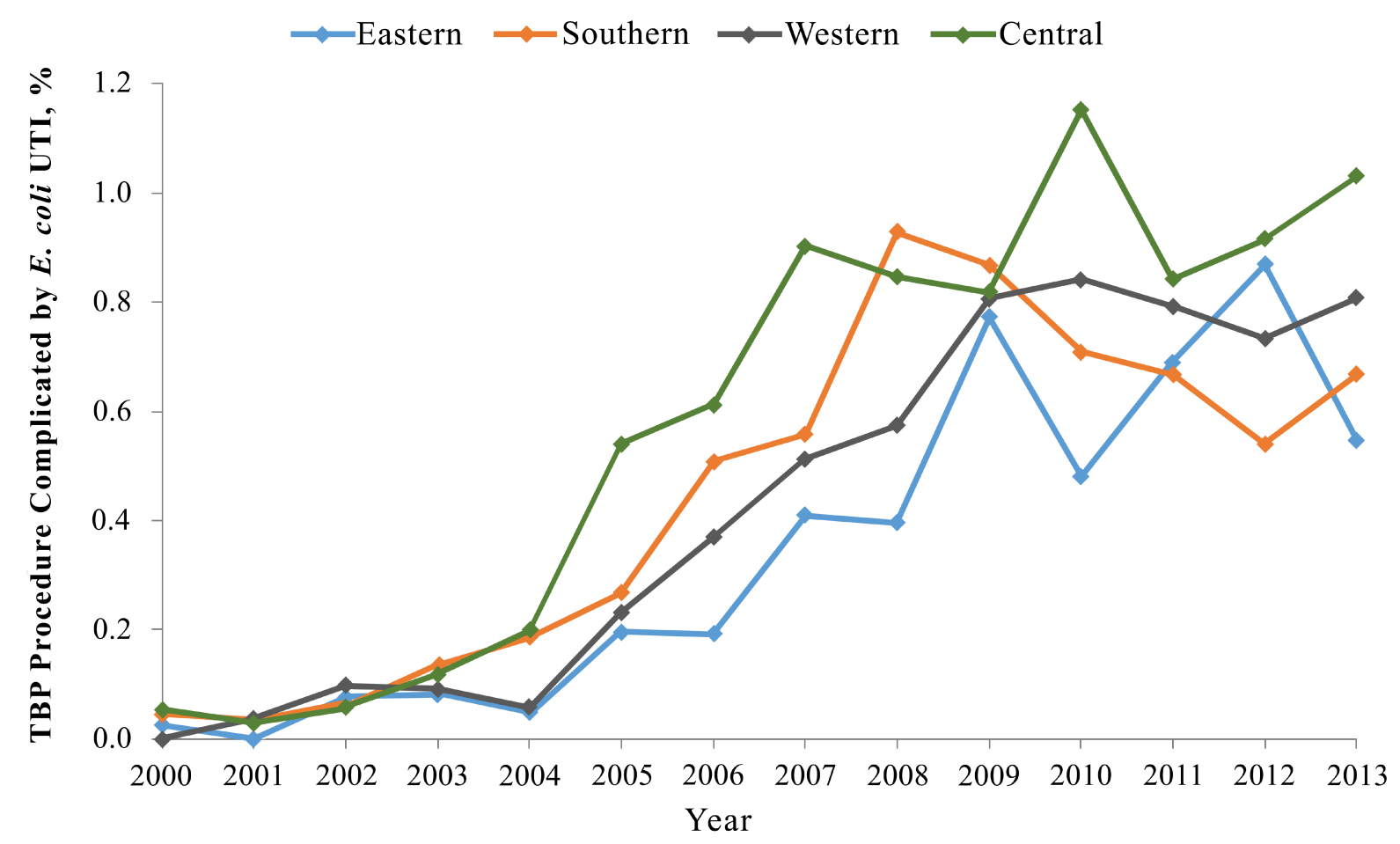

Figure 2. Escherichia coli Urinary Tract Infections Following Transrectal Biopsy of the Prostate, by Geographic Region

\section{Correlation of Prevalence of Fluoroquinolone-Resistance in all E. coli Isolates with Incidence of Post- TBP Infection due to Fluoroquinolone-Resistant E. coli by Facility}

Figure 3 shows the facility-level incidence of post-TBP infection with fluoroquinolone-resistant E. coli between 2008 and 2013, stratified by the prevalence of fluoroquinolone-resistance in all urinary E. coli isolates. The incidence of post-TBP infection with fluoroquinolone-resistant E. coli increased in a stepwise fashion as the prevalence of fluoroquinolone-resistance in all urinary $E$. coli isolates increased. Facilities with $<20 \%$ fluoroquinolone-resistance in urinary E. coli isolates had a 3-fold lower incidence of post-TBP infection with fluoroquinolone-resistant $E$. coli than facilities with $>40 \%$ fluoroquinolone-resistance in urinary $E$. coli isolates.

\section{Risk Factors for Infection with Fluoroquinolone-Resistant E. coli After TBP}

Between 2011 and 2013, a total of 59,613 patients underwent 67,183 TBP procedures. Table 1 shows a comparison of the characteristics of the 428 patients who developed post-TBP bacteriuria with fluoroquinolone-resistant $E$. coli and the 59,041 who did not develop any post-TPB bacteriuria infection with $E$. coli and odds ratios for development of infection. On univariable analysis, hospital admission in the previous year, isolation of a fluoroquinolone-resistant gram-negative bacillus in the previous year, diabetes mellitus, fluoroquinolone exposure in the previous year, and exposure to nonfluoroquinolone antibiotics in the previous year were significantly associated with post-TBP bacteriuria with fluoroquinolone-resistant E. coli. In the multivariable model, previous hospital admission, diabetes mellitus, previous isolation of a fluoroquinolone-resistant gram-neg- 
ative bacillus, and previous exposure to a fluoroquinolone remained significant as independent predictors of infection.

Although fluoroquinolone exposure was a significant risk factor for post-TBP bacteriuria with fluoroquinolone-resistant $E$. coli, only $27.6 \%$ of infected patients had received a fluoroquinolone in the year prior to the biopsy, excluding the 90 days prior to the procedure. In addition, only $15.7 \%$ of those with post-TBP bacteriuria with fluoroquinolone-resistant $E$. coli had been admitted to the hospital in the year before the procedure. Moreover, 169 (39.5\%) of the 428 patients who developed post-TBP bacteriuria with fluoroquinolone-resistant $E$. coli did not have any of the 4 characteristics identified as predictors of infection by the multivariable model.

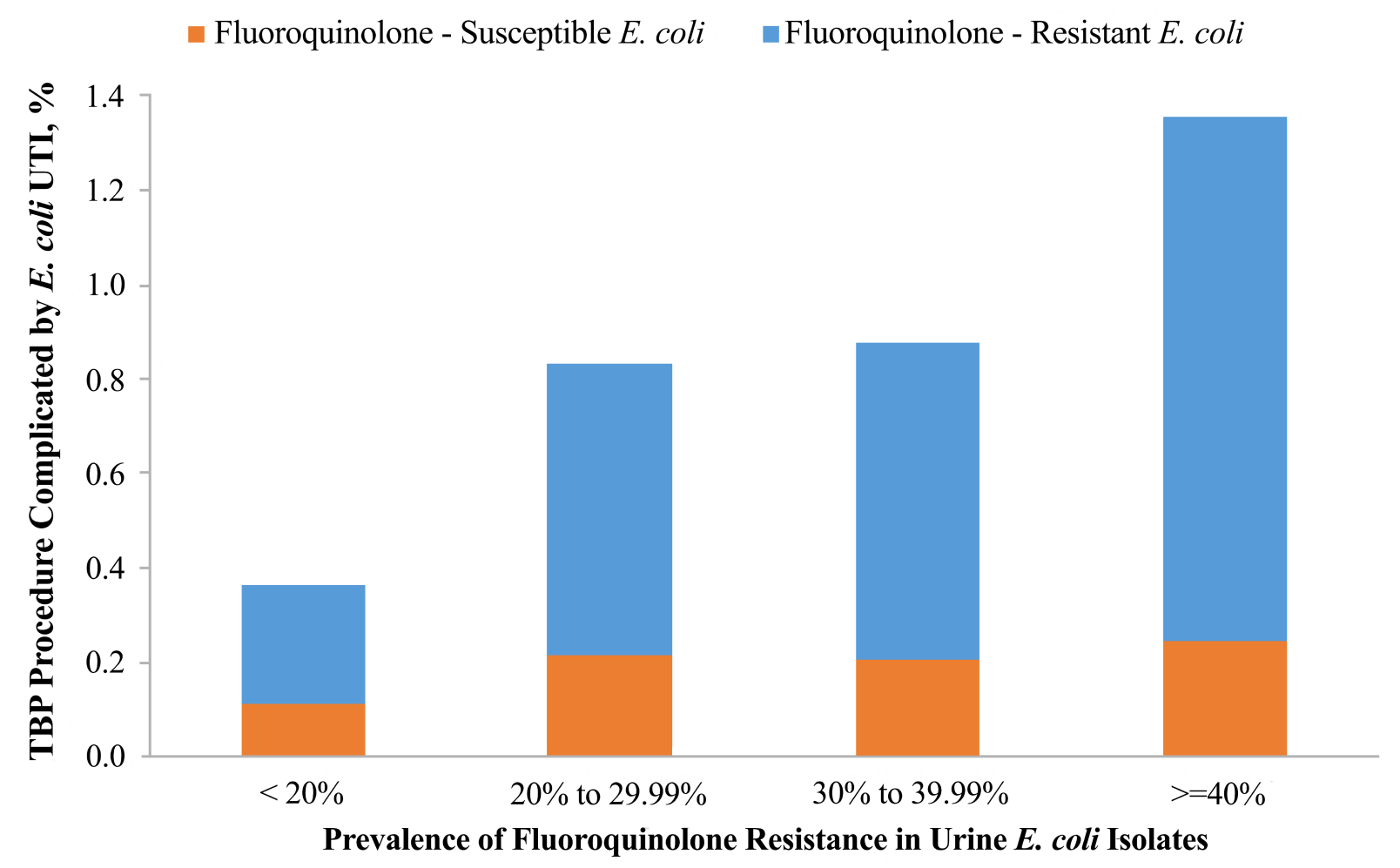

Figure 3. Facility Fluoroquinolone-Resistant Escherichia coli Urinary Tract Infections Following Transrectal Biopsy of the Prostate and Fluoroquinolone Resistance in All Urinary E. coli

\section{DISCUSSION}

In this national cohort study of veterans undergoing TBP, we found that there was a 5 -fold increase in the incidence of post-procedure E. coli bacteriuria and bacteremia between 2000 and 2013. The increase occurred in all regions of the country and was attributable to a dramatic increase in infections due to fluoroquinolone-resistant $E$. coli strains. The increasing incidence of fluoroquinolone-resistant $E$. coli infections after TBP occurred in parallel with increasing rates of fluoroquinolone-resistance in all urine and blood E. coli isolates. These findings build upon previous reports of increasing rates of post-TBP infections in individual hospitals or in multiple 
hospitals in a locality and suggest that there is an urgent need for development of effective strategies to prevent post-TBP infections.

Our findings suggest that the burden of post-TBP infections is significant in the United States. In the VA population, $0.9 \%$ of all TBP procedures in 2013 were complicated by E. coli bacteriuria and $0.2 \%$ by bacteremia; approximately $80 \%$ of these infections are due to fluoroquinolone-resistant E. coli isolates. If these frequencies are applicable to the approximately 1 million TBP procedures performed in the general United States population each year, an estimated 9000 E. coli UTIs and 2000 E. coli bloodstream infections occur yearly as a complication of TBP; more than 7200 of the UTIs and 1600 of the bloodstream infections are due to fluoroquinolone-resistant $E$. coli isolates. These infections may be associated with significant morbidity, costs, and frequent need for hospital admission [15-19]. For example, in a recent report, 51\% of patients with post-TBP infection due to fluoroquinolone-resistant gram-negative bacilli required hospital admission, 20\% had bacteremia, and $3 \%$ had abscess of the prostate [18].

Our findings have several important clinical implications. First, we identified several independent risk factors for fluoroquinolone-resistant E. coli bacteriuria after TBP that could be useful to identify a subset of patients at high risk for infection. These included diabetes mellitus, fluoroquinolone exposure, prior hospitalization, and prior cultures with fluoroquinolone-resistant gram-negative bacilli. However, it is notable that only $27.6 \%$ of those with fluoroquinolone-resistant E. coli infections had previous fluoroquinolone exposure. Moreover, 39.5\% of patients who developed post-TBP bacteriuria with fluoroquinolone-resistant $E$. coli did not have any of the 4 characteristics identified as independent predictors of infection. Second, the incidence of post-TBP infection with fluoroquinolone-resistant $E$. coli increased in a stepwise fashion as the prevalence of fluoroquinolone-resistance in all urinary $E$. coli isolates increased. Thus, monitoring of the prevalence of fluoroquinolone resistance in urinary E. coli isolates may provide a useful means to predict risk for post-TBP infections in a facility. Finally, many infection control programs do not conduct routine surveillance for infectious complications after outpatient biopsy procedures because the risk of infection is perceived to be low. Given the increasing prevalence of fluoroquinolone resistance in $E$. coli throughout the United States, routine surveillance for infections after TBP is indicated, particularly when rates of fluoroquinolone resistance are high in urinary E. coli isolates.

One notable observation from our study is that the rise in the incidence of $E$. coli infection after TBP peaked in 2008-2012, and has subsequently plateaued. One potential explanation for this observation might be that many urologists may have begun to implement interventions during this time period in response to rising rates of infection. As noted previously, modification of antimicrobial prophylaxis based on pre-procedure rectal culture results and modification of prophylaxis for all patients undergoing TBP have implemented in response to outbreaks, and have been associated with reductions in infection due to fluoroquinolone-resistant E. coli after TBP [20-25]. Future studies are needed to determine how widely disseminated such interventions have become. However, although changing strategies for prophylaxis may contribute to the recent reductions in post-TBP infection, the percentage of all E. coli urinary and blood isolates that are resistant to fluoroquinolones also has plateaued in the VA system. Thus, other factors may be contributing to the plateau in the incidence of infection after TBP. 
There were a number of limitations in this study. First, the study was conducted in the VA healthcare system and may not be generalizable to to US population at large. Second, it is possible that we underestimated the number of infections post-TBP because some patients may have sought care for post-TBP infections outside the VA system. Similarly, it is possible we underestimated antibiotic exposures because prescriptions outside the VA system were not captured. Third, we used bacteriuria as a surrogate for urinary tract infection, which would overestimate the incidence of $E$ coli infection; additionally, it is not known if bacteriuria with fluoroquinolone-resitant versus susceptible $E$ coli leads to different rates of symptomtic urinary tract infection. However, similar trends were observed for bacteremia isolates. Fourth, because fluoroquinolones for TBP prophylaxis are often prescribed during office visits in advance of the procedure, we excluded fluoroquinolone prescriptions in the 90 days prior to the procedure for the analysis of prior fluoroquinolone exposure. Thus, it is possible that we underestimated fluoroquinolone exposures if these agents were prescribed for other reasons in the 90 days before the procedure. Fifth, it is possible that we did not capture all TBP procedures if the procedure codes for transrectal ultrasound or ultrasonic guidance for the needle biopsy of the prostate were not coded for patients undergoing biopsy of the prostate. The transrectal approach is the predominant approach for prostate biopsy, and it has been demonstrated that use of procedural codes indicating ultrasound guidance may lead to decreased capture of qualifying procedures given the underutilization of these codes. Additionally, since medical record review for individual patients was not feasible, our analysis did not include or adjust for potentially important confounders such as case mix, duration of antibiotic exposure, and urologic abnormalities that might increase the risk for infection. Finally, our case-control approach is considered exploratory; a more refined analysis would include additional variables of interest, such as other comorbidities besides diabetes mellitus, as well as a more discerning antibiotic exposure review, and a comparison to similar susceptible infections.

\section{CONCLUSIONS}

In this national cohort of veterans undergoing TBP from 2000 through 2013, there was a 5-fold increase in post-procedure E. coli bacteriuria and a 4 -fold increase in post-procedure E. coli bacteremia that was attributable to a dramatic increase in infections due to fluoroquinolone-resistant E. coli and that occurred in parallel with increasing rates of fluoroquinolone-resistance in all urine and blood E. coli isolates. Our findings suggest that routine surveillance for postprocedure infections is indicated in all healthcare facilties performing TBP. In addition, studies are needed to identify effective strategies to prevent post-TBP infections.

\section{ACKNOWLEDGMENTS}

We thank the Veterans Informatics and Computing Infrastructure staff for assistance in obtaining the data and Makoto Jones, MD, and Siran Koroukian, PhD, for helpful discussions and critical review of the manuscript.

\section{POTENTIAL CONFLICTS OF INTEREST}

All authors have completed and submitted the ICMJE Form for Disclosure of Potential Financial Conflicts of Interest. Dr. Donskey is a member of Advisory Boards for Merck and Cubist and has received research funding from Merck and Cubist. No other disclosures were reported. 


\section{FINANCIAL SUPPORT}

This work was supported by the Department of Veterans Affairs and by the Cleveland VA Geriatric Research Education and Clinical Center. The investigators were responsible for the design and conduct of the study; collection, management, analysis, and interpretation of the data; preparation, review, and approval of the manuscript; and decision to submit the manuscript for publication. The funding agency was not involved in the design, execution, analysis, interpretation, or manuscript writing or critique. 
TABLE 1.

Risk Factors for Fluoroquinolone-Resistant Escherichia coli Bacteriuria After Transrectal Biopsy of the Prostate

\begin{tabular}{|c|c|c|c|c|c|c|}
\hline \multirow[t]{2}{*}{ Variable } & \multirow{2}{*}{$\begin{array}{l}\text { Control } \\
(\mathrm{N}=59,041)\end{array}$} & \multirow{2}{*}{$\begin{array}{l}\text { FQ-Resistant } E \text {. coli } \\
(\mathrm{N}=428)\end{array}$} & \multicolumn{2}{|c|}{ Univariable analysis } & \multicolumn{2}{|c|}{ Multivariable analysis } \\
\hline & & & $\begin{array}{l}\text { OR }(95 \% \\
\text { CI) }\end{array}$ & $\begin{array}{l}P \\
\text { Value }\end{array}$ & $\begin{array}{l}\text { OR }(95 \% \\
\text { CI) }\end{array}$ & $\begin{array}{l}P \\
\text { Value }\end{array}$ \\
\hline Age, median (range) & $65.0(44-93)$ & $64.9(25-97)$ & & 0.68 & & \\
\hline Diabetes mellitus & $16,940(28.7)$ & $168(39.3)$ & $\begin{array}{l}1.6(1.3- \\
2.0)\end{array}$ & $<0.0001$ & $\begin{array}{l}1.5(1.2- \\
1.8)\end{array}$ & $<0.0001$ \\
\hline $\begin{array}{l}\text { History of a culture positive } \\
\text { for FQ-resistant } E \text {. coli }\end{array}$ & $283(0.5)$ & $21(4.9)$ & $\begin{array}{l}10.7(6.8- \\
16.9)\end{array}$ & $<0.0001$ & $\begin{array}{l}7.4(4.6- \\
11.8)\end{array}$ & $<0.0001$ \\
\hline Admission in past year & $5,013(8.5)$ & $67(15.7)$ & $\begin{array}{l}2.0(1.5- \\
2.6)\end{array}$ & $<0.0001$ & $\begin{array}{l}1.5(1.2- \\
2.0)\end{array}$ & 0.003 \\
\hline $\begin{array}{l}\text { Fluoroquinolone use in the } \\
\text { past year }\end{array}$ & $11,537(19.7)$ & $122(27.6)$ & $\begin{array}{l}1.6(1.3- \\
2.0)\end{array}$ & $<0.0001$ & $\begin{array}{l}1.4(1.1- \\
1.7)\end{array}$ & 0.003 \\
\hline $\begin{array}{l}\text { Other antibiotic use in the } \\
\text { past year }^{\mathrm{a}}\end{array}$ & $16,883(28.6)$ & $153(35.8)$ & $\begin{array}{l}1.4(1.1- \\
1.7)\end{array}$ & 0.001 & $\begin{array}{l}1.1(0.90- \\
1.4)\end{array}$ & 0.27 \\
\hline
\end{tabular}

Abbreviations: CI: confidence interval; FQ: fluoroquinolone; OR: odds ratio.

Note: Data are number (\%) of patients unless otherwise specified.

${ }^{a}$ Included all identified systemic formulations of penicillins, cephalosporins, macrolides, trimethoprim-sulfamethoxazole, tetracyclines, and aminoglycosides. 


\section{REFERENCES}

1. Loeb S, Carter HB, Berndt SI, Ricker W, Schaeffer EM. Complications after prostate biopsy: data from SEER-Medicare. J Urol. 2011;186(5):1830-4. PubMed PMID: 21944136. doi: 10.1016/j.juro.2011.06.057

2. Liss MA, Johnson JR, Porter SB, Johnston B, Clabots C, Gillis K, Nseyo U, Holden M, Sakamoto K, Fierer J. Clinical and microbiological determinants of infection after transrectal prostate biopsy. Clin Infect Dis. 2015;60(7):979-87. PubMed PMID: 25516194. doi: 10.1093/cid/ciu1129

3. Rietbergen JB, Kruger AE, Kranse R, Schroder FH. Complications of transrectal ultrasound-guided systematic sextant biopsies of the prostate: evaluation of complication rates and risk factors within a population-based screening program. Urology. 1997;49(6):875-80. PubMed PMID: 9187694.

4. Otrock ZK, Oghlakian GO, Salamoun MM, Haddad M, Bizri ARN. Incidence of urinary tract infection following transrectal ultrasound guided prostate biopsy at a tertiary-care medical center in Lebanon. Infect Control Hosp Epidemiol. 2004;25(10):873-7. PubMed PMID: 15518032. doi: 10.1086/502312

5. Shigehara K, Miyagi T, Nakashima T, Shimamura M. Acute bacterial prostatitis after transrectal prostate needle biopsy: clinical analysis. J Infect Chemother Off J Japan Soc Chemother. 2008;14(1):40-3. PubMed PMID: 18297448. doi: 10.1007/s10156-0070570-3

6. Kim SJ, Kim S Il, Ahn HS, Choi JB, Kim YS, Kim SJ. Risk factors for acute prostatitis after transrectal biopsy of the prostate. Korean J Urol. 2010;51(6):426-30. PubMed PMID: 20577611. Pubmed Central PMCID: 2890061. doi: 10.4111/kju.2010.51.6.426

7. Hadway P, Barrett LK, Waghorn DJ, Hasan K, Bdesha A, Haldar N, Kelleher J. Urosepsis and bacteraemia caused by antibiotic-resistant organisms after transrectal ultrasonography-guided prostate biopsy. BJU Int. 2009;104(11):1556-8. PubMed PMID: 20053186. doi: 10.1111/j.1464-410X.2009.08959.x

8. Zaytoun OM, Vargo EH, Rajan R, Berglund R, Gordon S, Jones JS. Emergence of fluoroquinolone-resistant Escherichia coli as cause of postprostate biopsy infection: implications for prophylaxis and treatment. Urology. 2011;77(5):1035-41. PubMed PMID: 21420152. doi: 10.1016/j.urology.2010.12.067

9. Mosharafa AA, Torky MH, El Said WM, Meshref A. Rising incidence of acute prostatitis following prostate biopsy: fluoroquinolone resistance and exposure is a significant risk factor. Urology. 2011;78(3):511-4. PubMed PMID: 21782225. doi: 10.1016/j. urology.2011.04.064

10. Hedelin H, Claesson BEB, Wilpart A. Febrile reactions after transrectal ultrasound-guided prostatic biopsy: a retrospective study. Scand J Urol Nephrol. 2011;45(6):393-6. PubMed PMID: 21679017. doi: 10.3109/00365599.2011.590996

11. Zani EL, Clark OAC, Rodrigues Netto NJ. Antibiotic prophylaxis for transrectal prostate biopsy. Cochrane Database Syst Rev. 2011;(5):CD006576. PubMed PMID: 21563156. doi: 10.1002/14651858.CD006576.pub2 
12. Wolf JSJ, Bennett CJ, Dmochowski RR, Hollenbeck BK, Pearle MS, Schaeffer AJ. Best practice policy statement on urologic surgery antimicrobial prophylaxis. J Urol. 2008;179(4):1379-90. PubMed PMID: 18280509. doi: 10.1016/j.juro.2008.01.068

13. Johnson L, Sabel A, Burman WJ, Everhart RM, Rome M, MacKenzie TD, Rozwadowski J, Mehler PS, Price CS. Emergence of fluoroquinolone resistance in outpatient urinary Escherichia coli isolates. Am J Med. 2008;121(10):876-84. PubMed PMID: 18823859. doi: 10.1016/j.amjmed.2008.04.039

14. Neuhauser MM, Weinstein RA, Rydman R, Danziger LH, Karam G, Quinn JP. Antibiotic resistance among gram-negative bacilli in US intensive care units: implications for fluoroquinolone use. JAMA. 2003;289(7):885-8. PubMed PMID: 12588273.

15. Feliciano J, Teper E, Ferrandino M, Macchia RJ, Blank W, Grunberger I, Colon I. The incidence of fluoroquinolone resistant infections after prostate biopsy--are fluoroquinolones still effective prophylaxis? J Urol. 2008;179(3):952-5; discussion 955. PubMed PMID: 18207185. doi: 10.1016/j.juro.2007.10.071

16. Young JL, Liss MA, Szabo RJ. Sepsis due to fluoroquinolone-resistant Escherichia coli after transrectal ultrasound-guided prostate needle biopsy. Urology. 2009;74(2):332-8. PubMed PMID: 19464041. doi: 10.1016/j.urology.2008.12.078

17. Liss MA, Chang A, Santos R, Nakama-Peeples A, Peterson EM, Osann K, Billimek J, Szabo RJ, Dash A. Prevalence and significance of fluoroquinolone resistant Escherichia coli in patients undergoing transrectal ultrasound guided prostate needle biopsy. J Urol. 2011;185(4):1283-8. PubMed PMID: 21334021. Pubmed Central PMCID: 4063558. doi: 10.1016/j.juro.2010.11.088

18. Dumford D 3rd, Suwantarat N, Bhasker V, Kundrapu S, Zabarsky TF, Drawz P, Zhu H, Donskey CJ. Outbreak of fluoroquinolone-resistant Escherichia coli infections after transrectal ultrasound-guided biopsy of the prostate. Infect Control Hosp Epidemiol. 2013;34(3):269-73. PubMed PMID: 23388361. doi: 10.1086/669512

19. Nam RK, Saskin R, Lee Y, Liu Y, Law C, Klotz LH, Loblaw DA, Trachtenberg J, Stanimirovic A, Simor AE, Seth A, Urbach DR, Narod SA. Increasing hospital admission rates for urological complications after transrectal ultrasound guided prostate biopsy. J Urol. 2010;183(3):963-8. PubMed PMID: 20089283. doi: 10.1016/j.juro.2009.11.043

20. Taylor AK, Zembower TR, Nadler RB, Scheetz MH, Cashy JP, Bowen D, Murphy AB, Dielubanza E, Schaeffer AJ. Targeted antimicrobial prophylaxis using rectal swab cultures in men undergoing transrectal ultrasound guided prostate biopsy is associated with reduced incidence of postoperative infectious complications and cost of care. J Urol. 2012;187(4):1275-9. PubMed PMID: 22341272. doi: 10.1016/j.juro.2011.11.115

21. Duplessis CA, Bavaro M, Simons MP, Marguet C, Santomauro M, Auge B, Collard DA, Fierer J, Lesperance J. Rectal cultures before transrectal ultrasound-guided prostate biopsy reduce post-prostatic biopsy infection rates. Urology. 2012;79(3):556-61. PubMed PMID: 22386395. doi: 10.1016/j.urology.2011.09.057

22. Suwantarat N, Dumford DM 3rd, Ponce-Terashima R, Kundrapu S, Zabarsky TF, Zhu $\mathrm{H}$, Donskey CJ. Modification of antimicrobial prophylaxis based on rectal culture results to prevent fluoroquinolone-resistant Escherichia coli infections after prostate 
biopsy. Infect Control Hosp Epidemiol. 2013;34(9):973-6. PubMed PMID: 23917913. doi: $10.1086 / 671734$

23. Ho HSS, Ng LG, Tan YH, Yeo M, Cheng CWS. Intramuscular gentamicin improves the efficacy of ciprofloxacin as an antibiotic prophylaxis for transrectal prostate biopsy. Ann Acad Med Singapore. 2009;38(3):212-6. PubMed PMID: 19347074. Available from: http://www.annals.edu.sg/pdf/38VolNo3Mar2009/V38N3p212.pdf

24. Madden T, Doble A, Aliyu SH, Neal DE. Infective complications after transrectal ultrasound-guided prostate biopsy following a new protocol for antibiotic prophylaxis aimed at reducing hospital-acquired infections. BJU Int. 2011;108(10):1597-602. PubMed PMID: 21592292. doi: 10.1111/j.1464-410X.2011.10160.x

25. Batura D, Rao GG, Bo Nielsen P, Charlett A. Adding amikacin to fluoroquinolone-based antimicrobial prophylaxis reduces prostate biopsy infection rates. BJU Int. 2011;107(5):760-4. PubMed PMID: 21029317. doi: 10.1111/j.1464-410X.2010.09715.x

26. Hori S, Sengupta A, Joannides A, Balogun-Ojuri B, Tilley R, McLoughlin J. Changing antibiotic prophylaxis for transrectal ultrasound-guided prostate biopsies: are we putting our patients at risk? BJU Int. 2010;106(9):1298-302; discussion 1302. PubMed PMID: 20518764. doi: 10.1111/j.1464-410X.2010.09416.x

27. Colpan A, Johnston B, Porter S, Clabots C, Anway R, Thao L, Kuskowski MA, Tchesnokova V, Sokurenko EV, Johnson JR, Investigators V. Escherichia coli sequence type 131 (ST131) subclone H30 as an emergent multidrug-resistant pathogen among US veterans. Clin Infect Dis. 2013;57(9):1256-65. PubMed PMID: 23926176. Pubmed Central PMCID: 3792724. doi: 10.1093/cid/cit503

28. Butt AA, McGinnis K, Rodriguez-Barradas MC, Crystal S, Simberkoff M, Goetz MB, Leaf D, Justice AC, Veterans Aging Cohort S. HIV infection and the risk of diabetes mellitus. AIDS. 2009;23(10):1227-34. PubMed PMID: 19444074. Pubmed Central PMCID: 2752953. doi: 10.1097/QAD.0b013e32832bd7af

\section{COPYRIGHT}

(C) Pathogens and Immunity 2018

This work is licensed under a Creative Commons Attribution 4.0 International License. To view a copy of this license, visit http://creativecommons.org/licenses/by/4.0/ 